\begin{tabular}{|c|c|}
\hline Title & Finite-Element A nalysis of Lamb Wave Scattering in an Elastic Plate W aveguide \\
\hline Author(s) & Koshiba, M.; Karakida, S.; Suzuki, M. \\
\hline Citation & IEEE Transactions on Sonics and UItrasonics, 31(1), $18-24$ \\
\hline Issue Date & 1984-01 \\
\hline Doc URL & http:/hdl .handle.net/2115/6067 \\
\hline Rights & $\begin{array}{l}\text { ( } 1984 \text { IEEE. Personal use of this material is permitted. However, permission to reprint/republish this material for } \\
\text { advertising or promotional purposes or for creating new collective works for resale or redistribution to servers or lists, } \\
\text { or to reuse any copyrighted component of this work in other works must be obtained from the IEEE." } \\
\text { IEEE,IEEE Transactions on Sonics and UItrasonics, volume 31, issue 1, 1984, page18-24 }\end{array}$ \\
\hline Tyре & article \\
\hline Note(URL) & http://eeexplore.ieee.org/xpl freeabs_al I.jsp?isnumber=32865\& arnumber=1539532\& count=15\& index=7 \\
\hline File Information & ITSU 31-1.pdf \\
\hline
\end{tabular}

Instructions for use 


\title{
Finite-Element Analysis of Lamb Wave Scattering in an Elastic Plate Waveguide
}

\author{
MASANORI KOSHIBA, SHOJI KARAKIDA, AND MICHIO SUZUKI, SENIOR MEMBER, IEEE
}

\begin{abstract}
A method of the solution of scattering of the fundamental symmetric Lamb wave in an elastic plate waveguide is described. The approach is a combination of the finite-element and the analytical method. The numerical examples on the scattering by a wedge-shaped internal crack and a wedge-shaped surface crack in a plate are given. The reflection coefficient of the internal crack is considerably influenced by the wedge apex angle and the resonance phenomena arise in the region of the crack. On the other hand, the reflection coefficient of the surface crack is not very much affected by the wedge apex angle. The validity of the method is confirmed by comparing numerical results for an infinitely thin internal crack normal to the surface of a plate with the results of the variational method.
\end{abstract}

\section{INTRODUCTION}

$\mathrm{T}$ HE scattering of guided modes by the discontinuities in an elastic waveguide is a basic problem in nondestructive evaluation and also has important implications with regard to electrical signal-processing functions [1] -[4], and the scattering of Lamb waves by cracks in an elastic plate has been investigated extensively [5]-[10]. Auld et al. have analyzed the scattering of Lamb waves by an infinitely thin crack normal to the surface of a plate using the variational method [5]-[7]. Rokhlin has analyzed the scattering of Lamb waves by an infinitely thin crack parallel to the surface of a plate using the modified Wiener-Hopf method [8] or the method

Manuscript received May 4, 1983; revised October 11, 1983.

The authors are with the Department of Electronic Engineering, Hokkaido University, Sapporo, 060, Japan. of multiple diffractions [9], [10]. Although these methods are useful for the discontinuities of regular shape, it seems to be difficult to apply these methods to those of arbitrary shape. Recently the numerical method based on the finite-element method has been developed for the analysis of the scattering of horizontally polarized shear (SH) waves with a single particle-velocity component parallel to the plate surface [11][13]. This approach is useful for the analysis of the scattering by the arbitrarily shaped discontinuities.

In this paper, this numerical approach based on the finiteelement method is extended to the analysis of the scattering of Lamb waves with two particle-velocity components, lying in the plane perpendicular to the plate surface. Numerical examples on the scattering by a wedge-shaped internal crack and a wedge-shaped surface crack in a plate are given. The reflection coefficient of the internal crack is considerably influenced by the wedge apex angle and the resonance phenomena arise in the region of the crack. On the other hand, the reflection coefficient of the surface crack is not very much affected by the wedge apex angle. The validity of the method is confirmed by comparing numerical results for an infinitely thin internal crack normal to the surface of a plate with the results of the variational method [7].

\section{BASIC EQUATIONS}

In order to minimize the details, we consider the symmetric Lamb wave excitation of a symmetric plate waveguide as shown in Fig. 1, where the boundaries $\Gamma_{1}$ and $\Gamma_{2}$ connect 


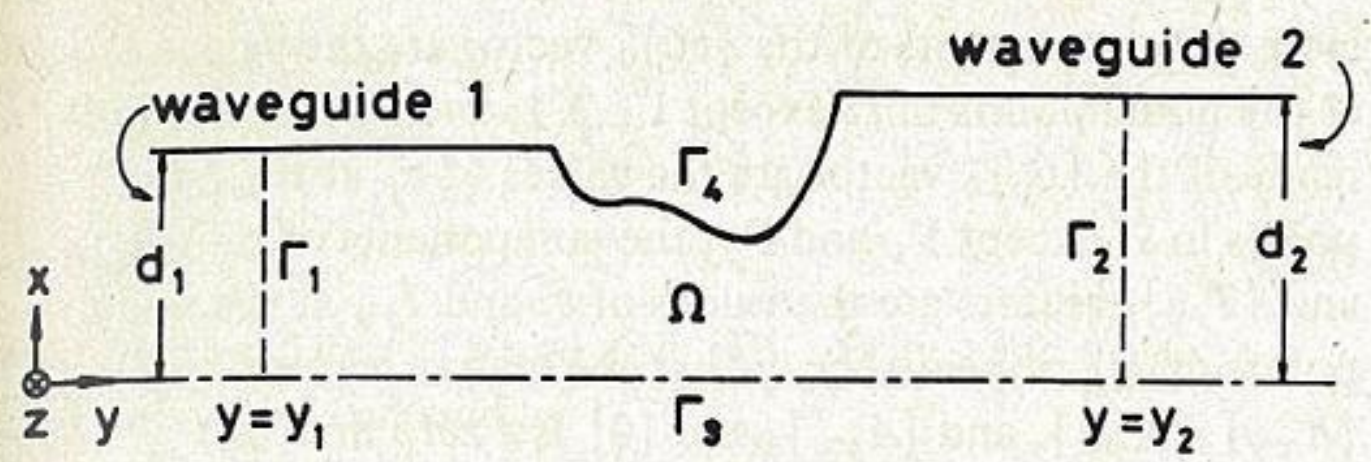

Fig. 1. Discontinuity in elastic plate waveguide.

the discontinuities to the waveguides 1 and 2, respectively, the $x$ component of the particle velocity $v_{x}$ and the $y$ component of the particle velocity $v_{y}$ are antisymmetric and symmetric about the midplane $\Gamma_{3}(x=0)$, respectively, the plane $\Gamma_{4}$ is the stress-free surface, and the region $\Omega$ with the boundaries $\Gamma_{1}$ to $\Gamma_{4}$ completely encloses the discontinuities. The plate waveguide is assumed to have some discontinuities which are symmetric about the midplane $\Gamma_{3}$.

Assuming that there is no variation in the $z$ direction, we have the following basic equations [1] :

$$
\begin{aligned}
& \partial T_{r x} / \partial x+\partial T_{r y} / \partial y-j \omega \rho v_{r}=0, \quad r=x, y \\
& {\left[\begin{array}{c}
j \omega T_{x x} \\
j \omega T_{y y} \\
j \omega T_{x y}
\end{array}\right]=\left[\begin{array}{ccc}
\lambda+2 \mu & \lambda & 0 \\
\lambda & \lambda+2 \mu & 0 \\
0 & 0 & \mu
\end{array}\right]\left[\begin{array}{c}
\partial v_{x} / \partial x \\
\partial v_{y} / \partial y \\
\partial v_{x} / \partial y+\partial v_{y} / \partial x
\end{array}\right]}
\end{aligned}
$$

where $T_{x x}, T_{y y}$, and $T_{x y}=T_{y x}$ are the stresses, $\omega$ is the angular frequency, $\rho$ is the mass density, and $\lambda$ and $\mu$ are the Lamé constants.

\section{Mathematical Formulation}

\section{A. Finite-Element Approach}

Dividing the region $\Omega$ into a number of second-order triangular elements [14], [15] as shown in Fig. 2, the particle velocity $v_{r}$ within each element is defined in terms of the particle velocity $v_{r, k}$ at the nodal point $k(k=1,2, \cdots, 6)$ :

$$
v_{r}=\{N\}^{T}\left\{v_{r}\right\}_{e}, \quad r=x, y,
$$

where

$$
\begin{aligned}
\left\{v_{r}\right\}_{e} & =\left[v_{r, 1} v_{r, 2} v_{r, 3} v_{r, 4} v_{r, 5} v_{r, 6}\right]^{T}, \quad r=x, y, \\
\{N\} & =\left[N_{1} N_{2} N_{3} N_{4} N_{5} N_{6}\right]^{T} .
\end{aligned}
$$

Here $\left\{v_{r}\right\}_{e}$ is the particle-velocity vector corresponding to the nodal points within each element, $T,\{\cdot\}$, and $\{\cdot\}^{T}$ denote a transpose, a column vector, and a row vector, respectively, and shape functions $N_{1}$ to $N_{6}$ are given by

$$
\begin{aligned}
& N_{1}=L_{1}\left(2 L_{1}-1\right) \\
& N_{2}=L_{2}\left(2 L_{2}-1\right) \\
& N_{3}=L_{3}\left(2 L_{3}-1\right) \\
& N_{4}=4 L_{1} L_{2} \\
& N_{5}=4 L_{2} L_{3} \\
& N_{6}=4 L_{3} L_{1}
\end{aligned}
$$

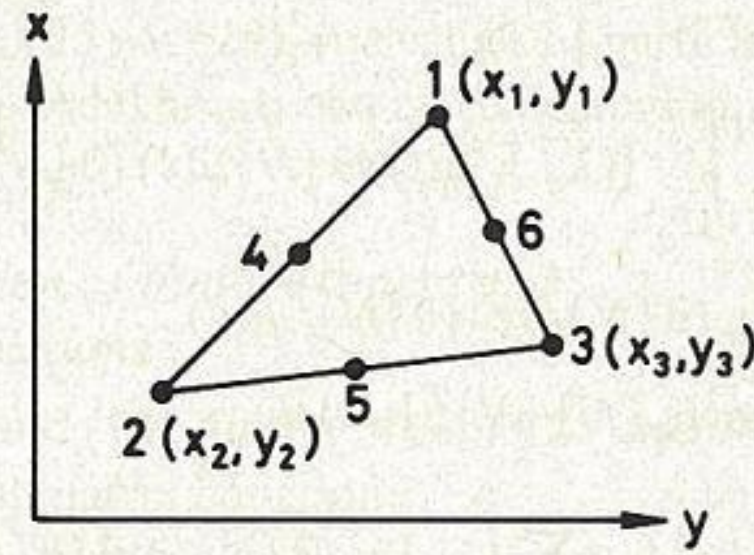

Fig. 2. Second-order triangular element.

with the area coordinates $L_{1}, L_{2}$, and $L_{3}[14]$, [15]. The relation equation between the area coordinates and Cartesian coordinates is given by

$$
\left[\begin{array}{l}
x \\
y \\
1
\end{array}\right]=\left[\begin{array}{ccc}
x_{1} & x_{2} & x_{3} \\
y_{1} & y_{2} & y_{3} \\
1 & 1 & 1
\end{array}\right]\left[\begin{array}{l}
L_{1} \\
L_{2} \\
L_{3}
\end{array}\right],
$$

where $\left(x_{j}, y_{j}\right)$ are the Cartesian coordinates of the vertex $j$ $(j=1,2,3)$ of the triangle.

Using a Galerkin procedure on (1), we obtain

$$
\begin{aligned}
& \int_{\Omega_{e}}\{N\}\left(\partial T_{r x} / \partial x+\partial T_{r y} / \partial y-j \omega \rho_{e} v_{r}\right) d \Omega=\{0\}, \\
& r=x, y,
\end{aligned}
$$

where $\Omega_{e}$ represents an element subdomain and $\{0\}$ is a null vector.

Integrating by parts, we obtain for (8)

$$
\begin{gathered}
\int_{\Omega_{e}}\left[(\partial\{N\} / \partial x) T_{r x}+(\partial\{N\} / \partial y) T_{r y}+j \omega \rho_{e}\{N\} v_{r}\right] d \Omega \\
-\int_{\Gamma_{e}}\{N\} T_{r n} d \Gamma=\{0\}, \quad r=x, y,
\end{gathered}
$$

where

$$
T_{r n}=T_{r x} n_{x}+T_{r y} n_{y}, \quad r=x, y .
$$

Here $\Gamma_{e}$ represents the contour of the triangular element, and $n_{x}$ and $n_{y}$ are the $x$ and $y$ components of an outward normal unit-vector to $\Gamma_{e}$, respectively.

Noting that $T_{x n}$ and $T_{y n}$ are continuous across $\Gamma_{e}$ (boundary conditions at the interface between two different media), $v_{x}=T_{y x}=0$ on $\Gamma_{3}$ (midplane), and $T_{x n}=T_{y n}=0$ on $\Gamma_{4}$ (stress-free surface), from (2), (3), and (9) the following global matrix equation is derived:

$$
\begin{aligned}
& {\left[\begin{array}{ll}
{\left[A_{x x}\right]} & {\left[A_{x y}\right]} \\
{\left[A_{y x}\right]} & {\left[A_{y y}\right]}
\end{array}\right]\left[\begin{array}{l}
\left\{v_{x}\right\} \\
\left\{v_{y}\right\}
\end{array}\right]} \\
& =\sum_{i=1}^{2} \sum_{e^{\prime}}(-1)^{i} j \omega\left[\begin{array}{l}
\int_{\Gamma_{i, e^{\prime}}}\{N\}_{i} T_{x y, i}\left(x, y_{i}\right) d x \\
\int_{\Gamma_{i, e^{\prime}}}\{N\}_{i} T_{y y, i}\left(x, y_{i}\right) d x
\end{array}\right]
\end{aligned}
$$


where

$$
\begin{aligned}
{\left[A_{x x}\right]=} & \sum_{e} \int_{\Omega_{e}}\left[\left(\lambda_{e}+2 \mu_{e}\right)(\partial\{N\} / \partial x)\left(\partial\{N\}^{T} / \partial x\right)\right. \\
& +\mu_{e}(\partial\{N\} / \partial y)\left(\partial\{N\}^{T} / \partial y\right) \\
& \left.-\omega^{2} \rho_{e}\{N\}\{N\}^{T}\right] d x d y \\
{\left[A_{x y}\right]=} & {\left[A_{y x}\right]^{T}=\sum_{e} \int_{\Omega_{e}}\left[\lambda_{e}(\partial\{N\} / \partial x)\left(\partial\{N\}^{T} / \partial y\right)\right.} \\
& \left.+\mu_{e}(\partial\{N\} / \partial y)\left(\partial\{N\}^{T} / \partial x\right)\right] d x d y \\
{\left[A_{y y}\right]=} & \sum_{e} \int_{\Omega_{e}}\left[\mu_{e}(\partial\{N\} / \partial x)\left(\partial\{N\}^{T} / \partial x\right)\right. \\
& +\left(\lambda_{e}+2 \mu_{e}\right)(\partial\{N\} / \partial y)\left(\partial\{N\}^{T} / \partial y\right) \\
& \left.-\omega^{2} \rho_{e}\{N\}\{N\}^{T}\right] d x d y .
\end{aligned}
$$

Here the components of the $\left\{v_{x}\right\}$ vector are the values of $v_{x}$ at all nodal points in $\Omega$ except $\Gamma_{3}$, the components of the $\left\{v_{y}\right\}$ vector are the values of $v_{y}$ at all nodal points in $\Omega, \Sigma_{e}$ and $\Sigma_{e^{\prime}}$ extend over all different elements and the elements related to $\Gamma_{i}(i=1,2)$, respectively, $T_{x y, i}\left(x, y_{i}\right)$ and $T_{y y, i}\left(x, y_{i}\right)$ are the stresses on $\Gamma_{i}$, and $\{N\}_{i}$ is the shape function vector on $\Gamma_{i}$, namely, $\{N\}_{i}=\left\{N\left(x, y_{i}\right)\right\}$.

Using the shape function $\{N\}_{i}$, the particle velocity $v_{r, i}\left(x, y_{i}\right)$ and the stress $T_{r y, i}\left(x, y_{i}\right)$ on $\Gamma_{i}$ may be discretized as follows:

$$
\begin{aligned}
v_{r, i}\left(x, y_{i}\right) & =\{N\}_{i}^{T}\left\{v_{r}\right\}_{i, e^{\prime}}, & & r=x, y, \\
T_{r y, i}\left(x, y_{i}\right) & =\{N\}_{i}^{T}\left\{T_{r y}\right\}_{i, e^{\prime}}, & & r=x, y,
\end{aligned}
$$

where $\left\{v_{r}\right\}_{i, e^{\prime}}$ and $\left\{T_{r y}\right\}_{i, e^{\prime}}$ are the particle-velocity vector and the stress vector corresponding to the nodal points within each element related to $\Gamma_{i}$, respectively.

Substituting (13) into (11), we obtain

$$
[A]\left[\begin{array}{c}
\left\{v_{x}\right\}_{0} \\
\left\{v_{y}\right\}_{0} \\
\left.\hdashline v_{x}\right\}_{12} \\
\left\{v_{y}\right\}_{12}
\end{array}\right]=\left[\begin{array}{c}
\{0\} \\
{[B]\left\{T_{x y}\right\}_{12}} \\
{[B]\left\{T_{y y}\right\}_{12}}
\end{array}\right],
$$

where

$$
\begin{aligned}
\left\{v_{r}\right\}_{12} & =\left[\begin{array}{l}
\left\{v_{r}\right\}_{1} \\
\left\{v_{r}\right\}_{2}
\end{array}\right], \quad r=x, y, \\
\left\{T_{r y}\right\}_{12} & =\left[\begin{array}{l}
\left\{T_{r y}\right\}_{1} \\
\left\{T_{r y}\right\}_{2}
\end{array}\right], \quad r=x, y, \\
{[B] } & =\left[\begin{array}{ll}
{[B]_{1}} & {[0]} \\
{[0]} & {[B]_{2}}
\end{array}\right],
\end{aligned}
$$

and

$$
[B]_{i}=(-1)^{i} j \omega \sum_{e^{\prime}} \int_{\Gamma_{i, e^{\prime}}}\{N\}_{i}\{N\}_{i}^{T} d x, \quad i=1,2
$$

Here the components of the $\left\{v_{x}\right\}_{0}$ vector are the values of $v_{x}$ at the nodal points in $\Omega$ except $\Gamma_{1}, \Gamma_{2}$, and $\Gamma_{3}$, the components of the $\left\{v_{y}\right\}_{0}$ vector are the values of $v_{y}$ at the nodal points in $\Omega$ except $\Gamma_{1}$ and $\Gamma_{2}$, the components of the $\left\{v_{r}\right\}_{i}$ and $\left\{T_{r y}\right\}_{i}$ vectors are the values of $v_{r}$ and $T_{r y}$ at the nodal points on $\Gamma_{i}$, respectively, $[A]$ is the matrix formed by $\left[A_{x x}\right]$, $\left[A_{x y}\right],\left[A_{y x}\right]$, and $\left[A_{y y}\right]$, and $[0]$ is a zero matrix.

\section{B. Analytical Approach}

In (14), the values of the stresses $T_{x y}$ and $T_{y y}$ on $\Gamma_{i}(i=1,2)$ are unknown, so that it is necessary to introduce the compatibility conditions for the interface boundary $\Gamma_{i}$ between the interior region $\left(y_{1} \leqq y \leqq y_{2}\right)$ and the exterior region $\left(y \leqq y_{1}\right.$, $\left.y \geqq y_{2}\right)$. Considering the analytical relation between $\left[\begin{array}{cc}-T_{x y} & v_{y}\end{array}\right]^{T}$ on $\Gamma_{i}$ and $\left[\begin{array}{ll}v_{x} & T_{y y}\end{array}\right]^{T}$ on $\Gamma_{i}$ which can be obtained by use of the orthogonality relation [1] , [2], [5] of the normal modes in an elastic plate waveguide, the values of particle velocities and stresses at the nodal points can be calculated numerically from (14). The solutions $\left[\begin{array}{ll}v_{x} & T_{y y}\end{array}\right]^{T}$ (or $\left[\begin{array}{ll}-T_{x y} & v_{y}\end{array}\right]^{T}$ ) on $\Gamma_{i}$ allow the determination of the reflection coefficient and the transmission coefficient. The details will be discussed later.

The internal fields in the waveguide $i(i=1,2)$ in Fig. 1 are represented by the sum of normal modes as [1], [2], [5]

$$
\begin{aligned}
{\left[\begin{array}{c}
v_{x, i}(x, y) \\
T_{y y, i}(x, y)
\end{array}\right]=} & \sum_{m}\left[a_{i m} \exp \left(-j \beta_{i m} y\right)+b_{i m} \exp \left(j \beta_{i m} y\right)\right] \\
& \cdot\left\{f_{i m}(x)\right\} \\
{\left[\begin{array}{c}
-T_{x y, i}(x, y) \\
v_{y, i}(x, y)
\end{array}\right]=} & \sum_{m}\left[a_{i m} \exp \left(-j \beta_{i m} y\right)-b_{i m} \exp \left(j \beta_{i m} y\right)\right] \\
& \cdot\left\{g_{i m}(x)\right\}
\end{aligned}
$$

where

$$
\left\{f_{i m}(x)\right\}=\left[f_{x, i m}(x) f_{y, i m}(x)\right]^{T}
$$

and

$$
\left\{g_{i m}(x)\right\}=\left[g_{x, i m}(x) g_{y, i m}(x)\right]^{T} .
$$

Here $a_{i m}$ and $b_{i m}$ are the amplitudes of the $m$ th mode ( $m=0,1,2, \cdots)$ in the waveguide $i$ propagating (or decaying) toward the $+y$ and $-y$ directions, respectively, and $\beta_{i m}$ is the wavenumber in the $y$ direction. The mode functions $\left\{f_{i m}(x)\right\}$ and $\left\{g_{i m}(x)\right\}$ satisfy the following orthonormalization condition [1]:

$$
\begin{aligned}
\int_{0}^{d_{i}}\left\{f_{i m}(x)\right\}^{T}\left\{g_{i m^{\prime}}(x)\right\} d x= & \int_{0}^{d_{i}}\left\{g_{i m}(x)\right\}^{T} \\
& \cdot\left\{f_{i m^{\prime}}(x)\right\} d x=\delta_{m m^{\prime}}
\end{aligned}
$$

where $\delta_{m m^{\prime}}$ is the Kronecker $\delta$. The dispersion relation for $\beta_{i m}$ and the mode functions are given in the Appendix.

Now, assuming that the fundamental Lamb wave $(m=0)$ of unit amplitude is incident from the left of waveguide 1 in Fig. 1, the amplitudes of normal modes may be written as 


$$
\begin{aligned}
& a_{1 m}= \begin{cases}1, & m=0 \\
0, & m=1,2,3, \cdots\end{cases} \\
& b_{2 m}=0 \quad m=0,1,2, \cdots
\end{aligned}
$$

Considering (19), (21), and (22), $T_{x y, i}\left(x, y_{i}\right)$ and $v_{y, i}\left(x, y_{i}\right)$ on $\Gamma_{i}$ may be expressed as

$$
\begin{aligned}
{\left[\begin{array}{c}
-T_{x y, i}\left(x, y_{i}\right) \\
v_{y, i}\left(x, y_{i}\right)
\end{array}\right]=} & 2 \delta_{i 1} \exp \left(-j \beta_{10} y_{1}\right)\left\{g_{10}(x)\right\} \\
& +(-1)^{i} \int_{0}^{d_{i}} \sum_{m}\left\{g_{i m}(x)\right\}\left\{g_{i m}\left(x^{\prime}\right)\right\}^{T} \\
& \cdot\left[\begin{array}{l}
v_{x, i}\left(x^{\prime}, y_{i}\right) \\
T_{y y, i}\left(x^{\prime}, y_{i}\right)
\end{array}\right] d x^{\prime} .
\end{aligned}
$$

Similarly, $v_{x, i}\left(x, y_{i}\right)$ and $T_{y y, i}\left(x, y_{i}\right)$ on $\Gamma_{i}$ may be expressed as

$$
\begin{aligned}
{\left[\begin{array}{c}
v_{x, i}\left(x, y_{i}\right) \\
T_{y y, i}\left(x, y_{i}\right)
\end{array}\right]=} & 2 \delta_{i 1} \exp \left(-j \beta_{10} y_{1}\right)\left\{f_{10}(x)\right\} \\
& +(-1)^{i} \int_{0}^{d_{i}} \sum_{m}\left\{f_{i m}(x)\right\}\left\{f_{i m}\left(x^{\prime}\right)\right\}^{T} \\
& \cdot\left[\begin{array}{c}
-T_{x y, i}\left(x^{\prime}, y_{i}\right) \\
v_{y, i}\left(x^{\prime}, y_{i}\right)
\end{array}\right] d x^{\prime}
\end{aligned}
$$

Using (3) and (13), (23) can be discretized as follows:

$$
\left[\begin{array}{c}
-\left\{T_{x y}\right\}_{i} \\
\left\{v_{y}\right\}_{i}
\end{array}\right]=\delta_{i 1}\left[\begin{array}{c}
\left\{g_{x}\right\}_{1} \\
\left\{g_{y}\right\}_{1}
\end{array}\right]+\left[\begin{array}{ll}
{\left[G_{x x}\right]_{i}} & {\left[G_{x y}\right]_{i}} \\
{\left[G_{y x}\right]_{i}} & {\left[G_{y y}\right]_{i}}
\end{array}\right]\left[\begin{array}{l}
\left\{v_{x}\right\}_{i} \\
\left\{T_{y y}\right\}_{i}
\end{array}\right]
$$

where

$$
\left\{g_{r}\right\}_{1}=2 \exp \left(-j \beta_{10} y_{1}\right)\left\{g_{r, 0}\right\}_{1}, \quad r=x, y,
$$

$\left[G_{r r^{\prime}}\right]_{i}=(-1)^{i} \sum_{m}\left\{g_{r, m}\right\}_{i} \sum_{e^{\prime}} \int_{\Gamma_{i, e^{\prime}}} g_{r^{\prime}, i m}\left(x^{\prime}\right)\left\{N\left(x^{\prime}, y_{i}\right)\right\}^{T} d x^{\prime}$, $r, r^{\prime}=x, y$.

Here the components of the $\left\{g_{r, m}\right\}_{i}$ vector are the values of $g_{r, i m}(x)$ at the nodal points on $\Gamma_{i}$.

Similarly, (24) can be discretized as follows:

$$
\left[\begin{array}{l}
\left\{v_{x}\right\}_{i} \\
\left\{T_{y y}\right\}_{i}
\end{array}\right]=\delta_{i 1}\left[\begin{array}{l}
\left\{f_{x}\right\}_{1} \\
\left\{f_{y}\right\}_{2}
\end{array}\right]+\left[\begin{array}{ll}
{\left[F_{x x}\right]_{i}} & {\left[F_{x y}\right]_{i}} \\
{\left[F_{y x}\right]_{i}} & {\left[F_{y y}\right]_{i}}
\end{array}\right]\left[\begin{array}{c}
-\left\{T_{x y}\right\}_{i} \\
\left\{v_{y}\right\}_{i}
\end{array}\right]
$$

where

$$
\begin{aligned}
&\left\{f_{r}\right\}_{1}=2 \exp \left(-j \beta_{10} y_{1}\right)\left\{f_{r, 0}\right\}_{1}, \quad r=x, y, \\
& {\left[F_{r r^{\prime}}\right]_{i} }=(-1)^{i} \sum_{m}\left\{f_{r, m}\right\}_{i} \sum_{e^{\prime}} \int_{\Gamma_{i, e^{\prime}}} f_{r^{\prime}, i m}\left(x^{\prime}\right)\left\{N\left(x^{\prime}, y_{i}\right)\right\}^{T} d x^{\prime}, \\
& r, r^{\prime}=x, y .
\end{aligned}
$$

Here the components of the $\left\{f_{r, m}\right\}_{i}$ vector are the values of $f_{r, i m}(x)$ at the nodal points on $\Gamma_{i}$.
Using either (25) or (28), the values of particle velocities and stresses at the nodal points can be calculated numerically from (14).

\section{Combination of Finite-Element and Analytical Relations}

Using (15) and (16), from (14) and (25) we obtain the following final matrix equation:

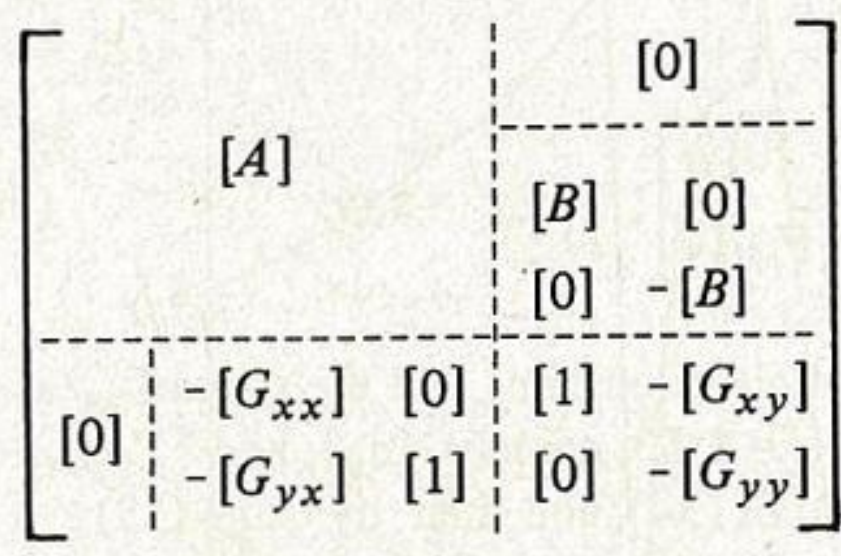

$$
\left[\begin{array}{c}
\left\{v_{x}\right\}_{0} \\
\left\{v_{y}\right\}_{0} \\
\hdashline\left\{v_{x}\right\}_{12} \\
\left\{v_{y}\right\}_{12} \\
\hdashline-\left\{T_{x y}\right\}_{12} \\
\left\{T_{y y}\right\}_{12}
\end{array}\right]=\left[\begin{array}{c}
\{0\} \\
-\{0\} \\
\left\{g_{x}\right\}_{12} \\
\left\{g_{y}\right\}_{12}
\end{array}\right]
$$

where

$$
\begin{aligned}
& \left\{g_{r}\right\}_{12}=\left[\begin{array}{c}
\left\{g_{r}\right\}_{1} \\
\{0\}
\end{array}\right], \quad r=x, y, \\
& {\left[G_{r r^{\prime}}\right]=\left[\begin{array}{cc}
{\left[G_{r r^{\prime}}\right]_{1}} & {[0]} \\
{[0]} & {\left[G_{r r^{\prime}}\right]_{2}}
\end{array}\right], \quad r, r^{\prime}=x, y .}
\end{aligned}
$$

Here [1] is a unit matrix.

Similarly, from (14) and (28) we obtain the following final matrix equation:

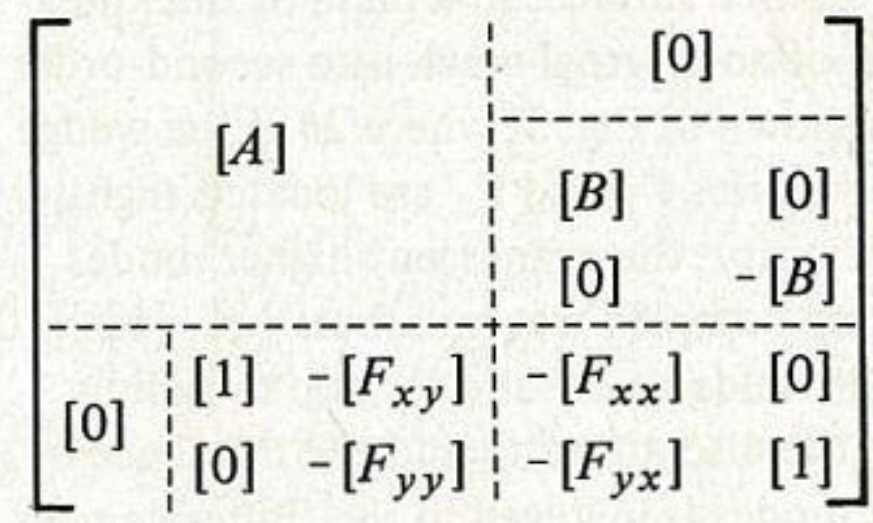

where

$$
\left[\begin{array}{c}
\left\{v_{x}\right\}_{0} \\
\left\{v_{y}\right\}_{0} \\
\hdashline\left\{v_{x}\right\}_{12} \\
\left\{v_{y}\right\}_{12} \\
\hdashline-\left\{T_{x y}\right\}_{12} \\
\left\{T_{y y}\right\}_{12}
\end{array}\right]=\left[\begin{array}{c}
\{0\} \\
\{0\} \\
\left.\hdashline f_{x}\right\}_{12} \\
\left\{f_{y}\right\}_{12}
\end{array}\right]
$$

$$
\left\{f_{r}\right\}_{12}=\left[\begin{array}{r}
\left\{f_{r}\right\}_{1} \\
\{0\}
\end{array}\right], \quad r=x, y
$$




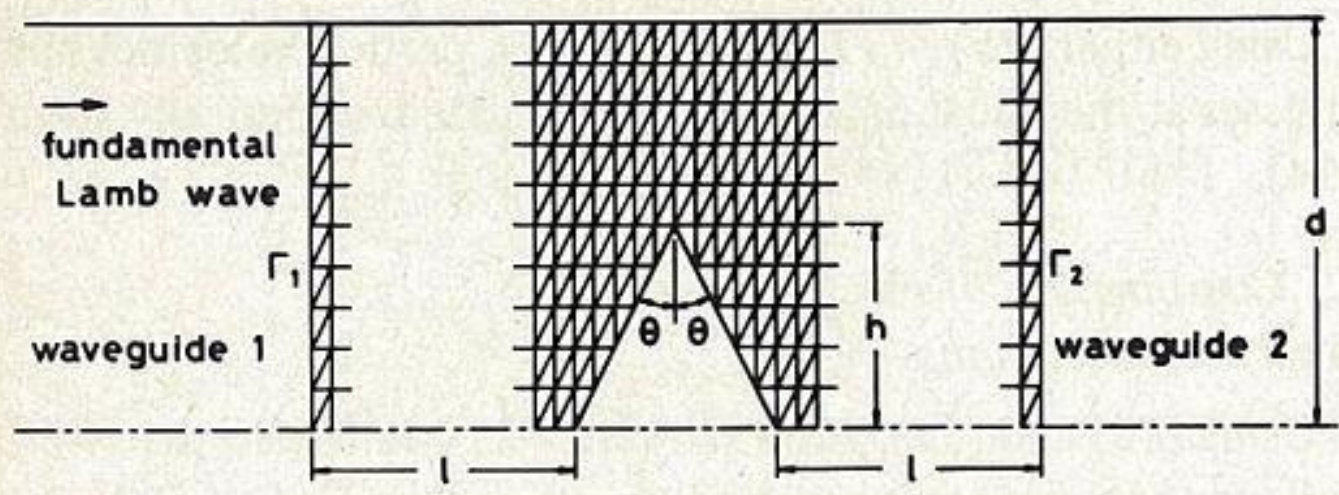

Fig. 3. Typical division of wedge-shaped internal crack.

$$
\left[F_{r r^{\prime}}\right]=\left[\begin{array}{cc}
{\left[F_{r r^{\prime}}\right]_{1}} & {[0]} \\
{[0]} & {\left[F_{r r^{\prime}}\right]_{2}}
\end{array}\right], \quad r, r^{\prime}=x, y .
$$

The values of the particle velocity $v_{x}$ and the stress $T_{y y}$ at the nodal points on $\Gamma_{i}(i=1,2)$, namely, $\left\{v_{x}\right\}_{i}$ and $\left\{T_{y y}\right\}_{i}$, are computed from (31) or (34), and then, by using (13), $v_{x, i}\left(x, y_{i}\right)$ and $T_{y y, i}\left(x, y_{i}\right)$ are calculated from these values. The solutions $v_{x, i}\left(x, y_{i}\right)$ and $T_{y y, i}\left(x, y_{i}\right)$ allow the determination of the reflection coefficient $r$ at $y=y_{1} \equiv 0$ and the transmission coefficient $t$ at $y=y_{2}$ of the fundamental Lamb wave. Considering (19), (21), and (22), $r$ and $t$ may be written as

$$
\begin{aligned}
& r \equiv b_{10}=-1+\int_{0}^{d_{1}}\left\{g_{10}(x)\right\}^{T}\left[\begin{array}{l}
v_{x, 1}\left(x, y_{1}=0\right) \\
T_{y y, 1}\left(x, y_{1}=0\right)
\end{array}\right] d x, \quad(37) \\
& t \equiv a_{20} \exp \left(-j \beta_{20} y_{2}\right)=\int_{0}^{d_{2}}\left\{g_{20}(x)\right\}^{T}\left[\begin{array}{l}
v_{x, 2}\left(x, y_{2}\right) \\
T_{y y, 2}\left(x, y_{2}\right)
\end{array}\right] d x .
\end{aligned}
$$

\section{Analysis of Lamb Wave Scattering BY A CRACK}

In this section, we present the computed results for the fundamental Lamb wave reflection coefficient of a wedgeshaped crack with stress-free surfaces in a plate of thickness $2 d$. A typical division of an internal crack into second-order triangular elements is shown in Fig. 3, where $2 \theta$ is the wedge apex angle. If the boundaries $\Gamma_{1}$ and $\Gamma_{2}$ are located slightly away from the discontinuity, the evanescent higher modes with pure imaginary and complex wavenumbers [1], [5] -[10] have little effect on the fields on $\Gamma_{1}$ and $\Gamma_{2}$ due to rapidly decaying modes from the discontinuity and the influence of the evanescent higher modes is involved in the finite-element region $\Omega$. Hence it is usually sufficient to consider the propagating modes only in (27) and (30), and, if necessary, a few evanescent higher modes for a reasonable approximation. Convergence of the solution is checked by increasing a value of $l$ in Fig. 3. In this calculation, a Poisson's ratio $\sigma=0.31$ and $2 k_{s} d / \pi=1.0$, where $k_{s}=\omega \sqrt{\rho / \mu}$. All the higher modes are nonpropagating evanescent modes. In order to present the numerical verification of the method, Fig. 4 shows the magnitude and the phase of the reflection coefficient of an infinitely thin internal crack $(\theta=0)$ normal to the surface of a plate as a function of $l / \lambda_{L 0}$, where $\lambda_{L 0}$ is the wavelength of the fundamental Lamb wave and the crack to plate thickness ratio $h / d=0.5$. The phase is evaluated at the position

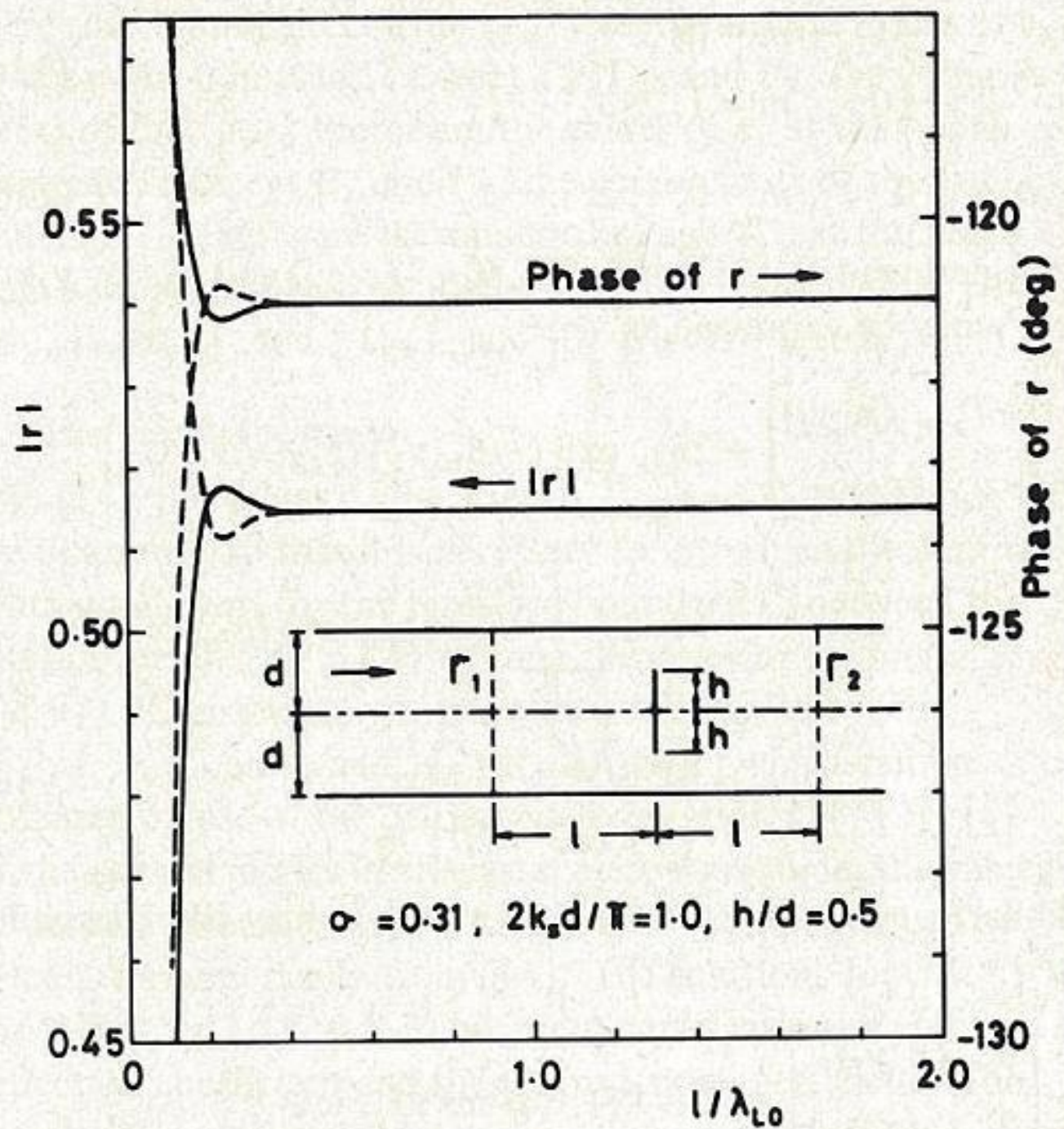

Fig. 4. Convergence behavior in calculation of reflection coefficients of an infinitely thin internal crack. The solid line is the results obtained by using (31). The broken line is the results obtained by using (34).

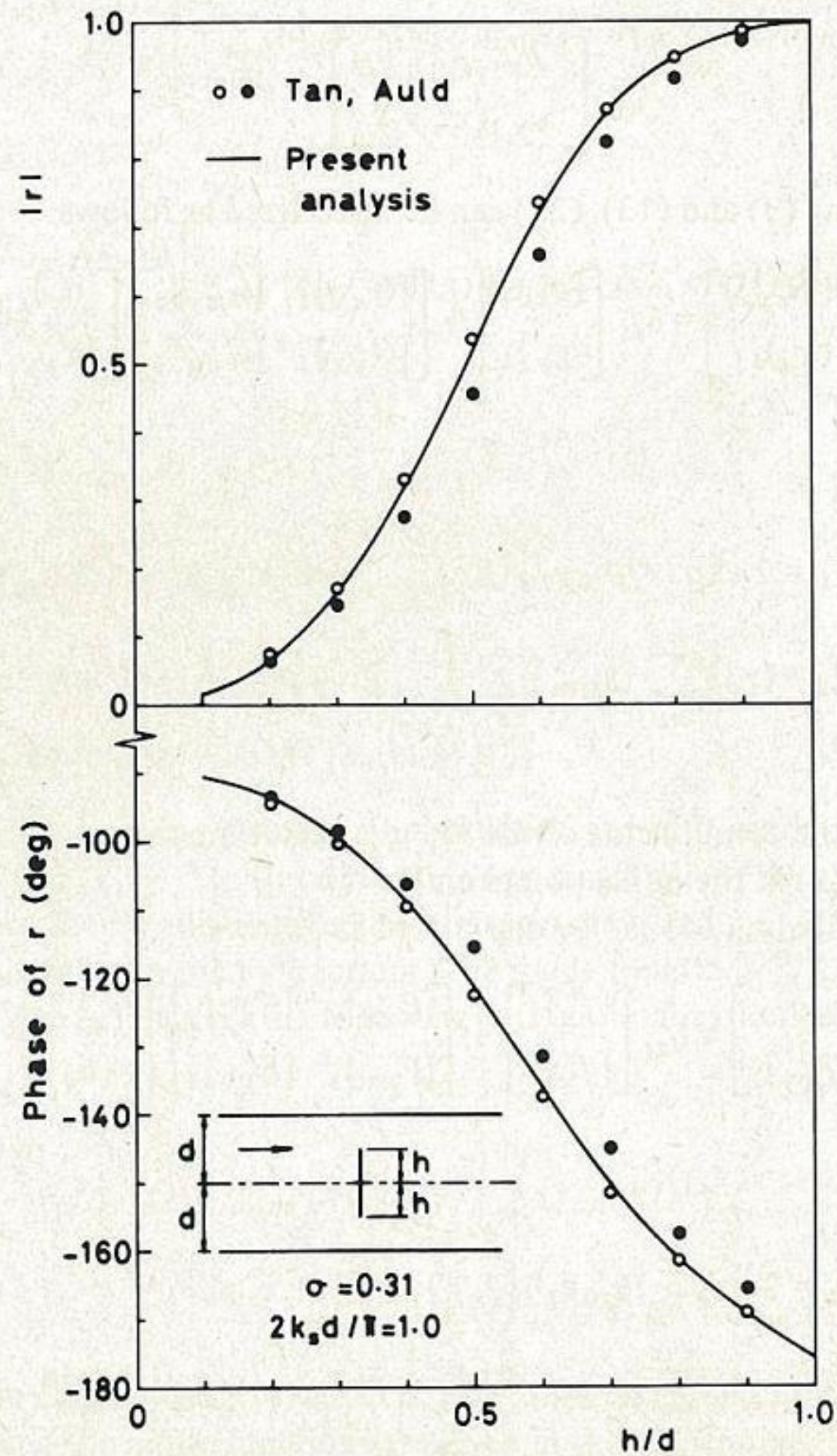

Fig. 5. Reflection coefficient of an infinitely thin internal crack. o results [7] of variational method with the trial function $v_{y}=$ $\sqrt{1-(x / h)^{2}}$ and $v_{x}=0$. results [7] of variational method with the trial function $v_{y}=\cos (\pi x / 2 h)$ and $v_{x}=0$. 


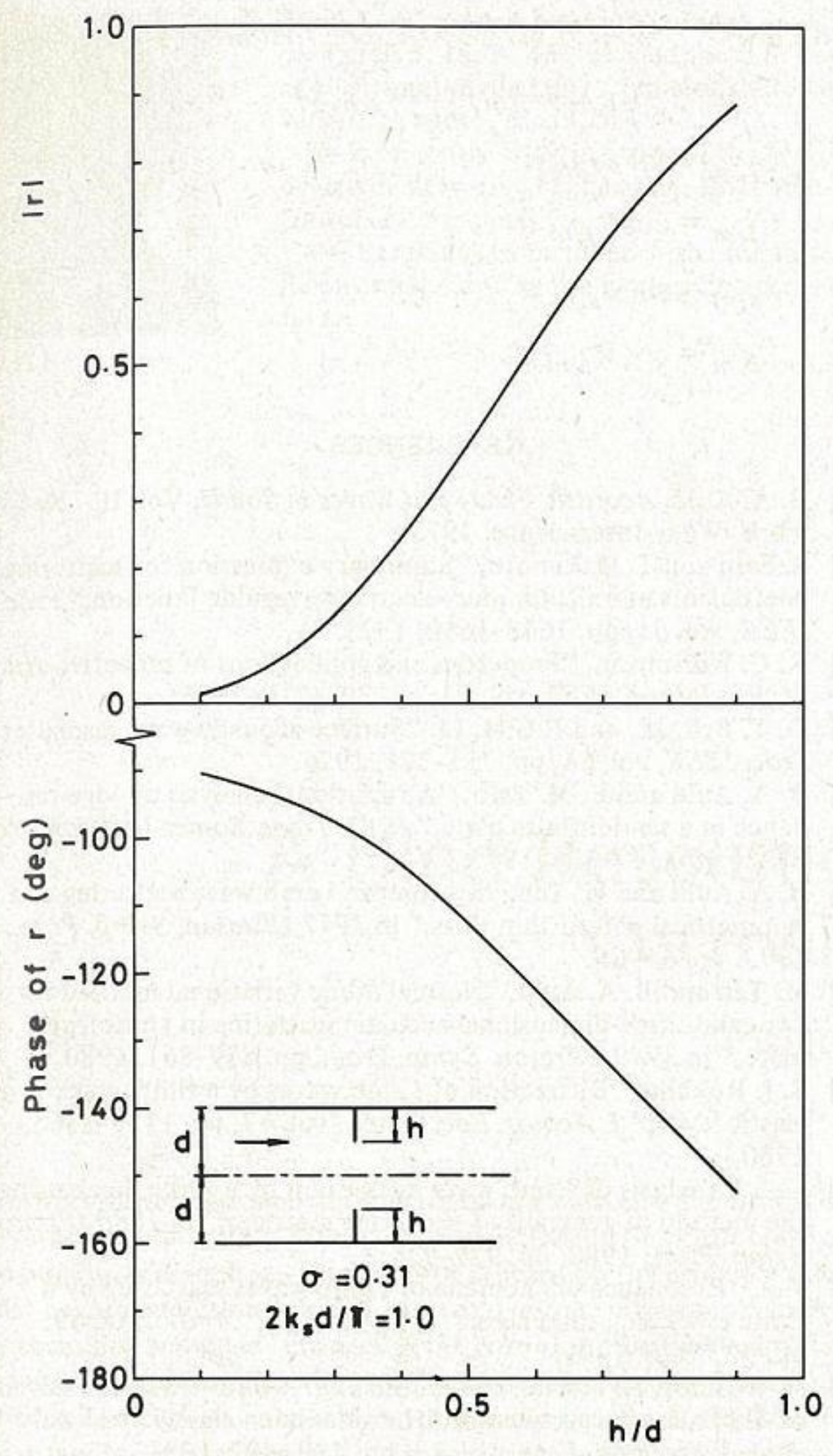

Fig. 6. Reflection coefficient of infinitely thin surface crack.

of a crack. The difference between the value (solid lines) calculated from (31) and the value (dashed lines) calculated from (34) can be distinguished only for the range of $l / \lambda_{L 0} \lesssim$ 0.5 , and the two values converge with $l / \lambda_{L 0} \simeq 0.5$. Therefore, we set $l$ equal to $2 \lambda_{L 0}$ and present only the results obtained by using (31).

Fig. 5 shows the magnitude and the phase of the reflection coefficient of an infinitely thin internal crack normal to the surface of a plate as a function of $h / d$. Agreement between our results and the results (circles) of the variational method with the trial function $v_{y}=\sqrt{1-(x / h)^{2}}$ and $v_{x}=0$ [7] is seen to be excellent. The results are in good agreement with the results (dots) of the variation method with the trial function $v_{y}=\cos (\pi x / 2 h)$ and $v_{x}=0[7]$ at small values of $h / d$.

Fig. 6 shows the magnitude and the phase of the reflection coefficient of an infinitely thin surface crack normal to the surface of a plate as a function of $h / d$. It is seen that the magnitude of the reflection coefficient of the infinitely thin surface crack is smaller than that of the infinitely thin internal crack.

Figs. 7 and 8 show the magnitude of the reflection coefficient of a wedge-shaped internal crack and a wedge-shaped surface crack as a function of the wedge apex angle $\tan \theta$,

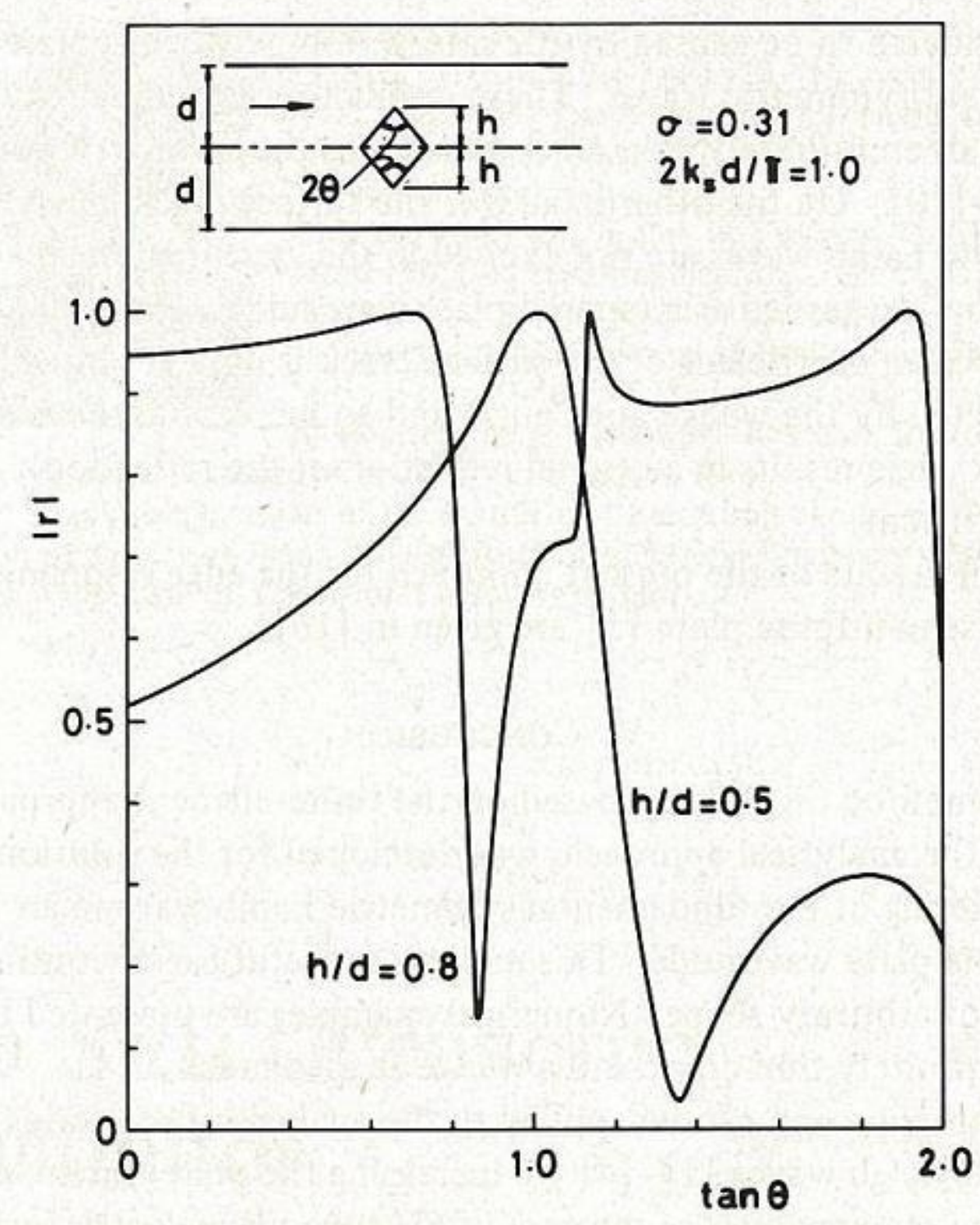

Fig. 7. Reflection coefficient of wedge-shaped internal crack.

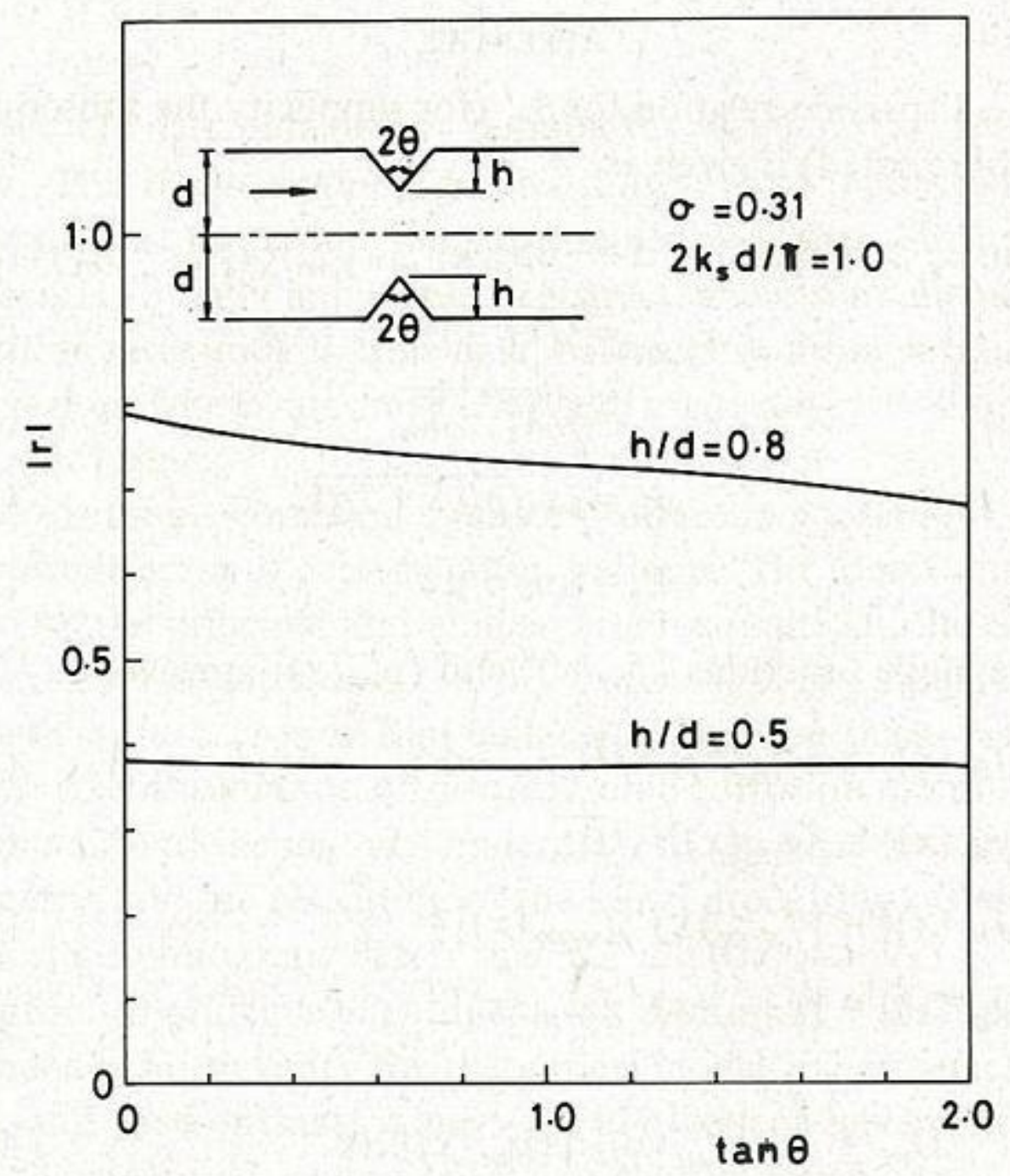

Fig. 8. Reflection coefficient of wedge-shaped surface crack.

respectively. The reflection coefficient of the internal crack is considerably influenced by the wedge apex angle and the curves exhibit resonances, resulting in complete reflection of the incident wave. By virtue of the problem's symmetry, for the incidence of the fundamental symmetric Lamb wave the antisymmetric Lamb waves are not excited in a plate except the region occupied by the crack. In the crack-region the plate waveguide branches into two waveguides, and both symmetric and antisymmetric Lamb waves are excited in each separated waveguide of length $2 h \tan \theta$. The resonances are 
considered to be caused by the interaction between symmetric and antisymmetric waves. These resonances are similar to the case of an infinitely thin crack parallel to the surface of a plate [8]-[10]. On the other hand, for the surface crack antisymmetric Lamb waves are not excited in the crack-region and this region is regarded as a tapered plate waveguide. Therefore, the reflection coefficient of the surface crack is not very much affected by the wedge apex angle and an increase in the wedge apex angle results in a general reduction of the reflection coefficient.

The results of the present approach for the edge resonance in a semi-infinite plate [5] are given in [16].

\section{CONCLUSION}

A method of analysis, based on the finite-element approach and the analytical approach, was developed for the solution of scattering of the fundamental symmetric Lamb wave in an elastic plate waveguide. This method is useful for discontinuities of arbitrary shape. Numerical examples are presented for an infinitely thin crack and a wedge-shaped crack.

This approach can be applied to the analysis of scattering of Rayleigh waves [1]- [4] by increasing the plate thickness in the same manner as the case of SH-type piezoelectric surface waves [12].

\section{APPENDIX}

The dispersion relation for $\beta_{m}$ (for simplicity the subscript $i$ is abbreviated) is given as

$$
\begin{aligned}
\tan \kappa_{s, m} d / \tan \kappa_{l, m} d & =-4 \beta_{m}^{2} \kappa_{l, m} \kappa_{s, m} /\left(\kappa_{s, m}^{2}-\beta_{m}^{2}\right) \\
\kappa_{l, m} & =\sqrt{k_{l}^{2}-\beta_{m}^{2}} \\
\kappa_{s, m} & =\sqrt{k_{s}^{2}-\beta_{m}^{2}} \\
k_{l} & =\omega \sqrt{\rho /(\lambda+2 \mu)} \\
k_{s} & =\omega \sqrt{\rho / \mu} .
\end{aligned}
$$

The mode functions $\left\{f_{m}(x)\right\}$ and $\left\{g_{m}(x)\right\}$ are given as

$$
\begin{aligned}
\left\{f_{m}(x)\right\} & =\left\{\bar{f}_{m}(x)\right\} / \sqrt{D_{m}} \\
\left\{g_{m}(x)\right\} & =\left\{\bar{g}_{m}(x)\right\} / \sqrt{D_{m}} \\
\left\{\bar{f}_{m}(x)\right\} & =\left[\bar{f}_{x, m}(x) \bar{f}_{y, m}(x)\right]^{T} \\
\left\{\bar{g}_{m}(x)\right\} & =\left[\bar{g}_{x, m}(x) \bar{g}_{y, m}(x)\right]^{T} \\
D_{m} & =\int_{0}^{d}\left\{\bar{f}_{m}(x)\right\}^{T}\left\{\bar{g}_{m}(x)\right\} d x
\end{aligned}
$$

$\bar{f}_{x, m}(x)=\sin \kappa_{l, m} x-\left[2 \beta_{m}^{2} /\left(\beta_{m}^{2}-\kappa_{s, m}^{2}\right)\right]$

$$
\cdot\left(S_{l, m} / S_{s, m}\right) \sin \kappa_{s, m} x
$$

$\bar{f}_{y, m}(x)=-j\left(\mu / \omega \kappa_{l, m}\right)\left[\left(k_{s}^{2}-2 \kappa_{l, m}^{2}\right)\right.$

$$
\begin{aligned}
& \cdot \cos \kappa_{l, m} x-\left(\beta_{m}^{2}-\kappa_{s, m}^{2}\right) \\
& \left.\cdot\left(C_{l, m} / C_{s, m}\right) \cos \kappa_{s, m} x\right]
\end{aligned}
$$

$\bar{g}_{x, m}(x)=\left(2 \mu \beta_{m} / \omega\right)\left[\sin \kappa_{l, m} x-\left(S_{l, m} / S_{s, m}\right) \sin \kappa_{s, m} x\right]$

$$
\begin{aligned}
\bar{g}_{y, m}(x)= & j\left(\beta_{m} / \kappa_{l, m}\right)\left(\cos \kappa_{l, m} x-\left[\left(\beta_{m}^{2}-\kappa_{s, m}^{2}\right) / 2 \beta_{m}^{2}\right]\right. \\
& \left.\cdot\left(C_{l, m} / C_{s, m}\right) \cos \kappa_{s, m} x\right) \\
S_{l, m}= & \sin \kappa_{l, m} d \\
C_{l, m}= & \cos \kappa_{l, m} d \\
S_{s, m}= & \sin \kappa_{s, m} d \\
C_{s, m}= & \cos \kappa_{s, m} d
\end{aligned}
$$

\section{REFERENCES}

[1] B. A. Auld, Acoustic Fields and Waves in Solids, Vol. II. New York: Wiley-Interscience, 1973.

[2] S. Sato and T. Makimoto, "Stationary expression for scattering coefficients at uniform piezoelectric waveguide junction," Proc. $I E E E$, vol. 61, pp. 1648-1650, 1973.

[3] R. C. Williamson, "Properties and applications of reflective-array devices," Proc. IEEE, vol. 64, pp. 702-710, 1976.

[4] D. T. Bell, Jr., and R.C.M. Li, "Surface-acoustic-wave resonator," Proc. IEEE, vol. 64, pp. 711-721, 1976.

[5] B. A. Auld and E. M. Tsao, "A variational analysis of edge resonance in a semi-infinite plate," IEEE Trans. Sonics Ultrason., vol. SU-24, pp. 317-326, 1977.

[6] B. A. Auld and M. Tan, "Symmetric Lamb wave scattering at a symmetrical pair of thin slots," in 1977 Ultrason. Symp. Proc., 1977, pp. 61-66.

[7] M. Tan and B. A. Auld, "Normal mode variational method for two- and three-dimensional acoustic scattering in an isotropic plate," in 1980 Ultrason. Symp. Proc., pp. 857-861, 1980.

[8] S. I. Rokhlin, "Diffraction of Lamb waves by a finite crack in an elastic layer," J. Acoust. Soc. Amer., vol. 67, pp. 1157-1165, 1980.

[9] -, "Analysis of Lamb wave diffraction by a finite crack using the method of generalized scattering matrices," in 1980 Ultrason. Symp. Proc., 1980, pp. 922-926.

[10] - "Resonance phenomena of Lamb waves scattering by a finite crack in a solid layer," J. Acoust. Soc. Amer., vol. 69, pp. 922-928, 1981.

[11] M. Koshiba, H. Morita, and M. Suzuki, "Finite-element analysis of discontinuity problem of SH modes in an elastic plate waveguide," Electron. Lett., vol. 17, pp. 480-482, 1981.

[12] _ "Finite-element analysis of discontinuity problem of SH-type modes in a piezoelectric plate waveguide," Trans. Inst. Electron. Commun. Eng. Japan, vol. E65, pp. 522-528, 1982.

[13] Z. Abduljabbar, S. K. Datta, and A. H. Shah, "Diffraction of horizontally polarized shear waves by normal edge cracks in a plate," J. Appl. Phys., vol. 52, pp. 461-472, 1983.

[14] O. C. Zienkiewicz, The Finite Element Method in Engineering Science. London: McGraw Hill, 1971.

[15] P. E. Lagasse, "Higher-order finite-element analysis of topographic guides supporting elastic surface waves," J. Acoust. Soc. Amer., vol. 53, pp. 1116-1122, 1973.

[16] M. Koshiba, S. Karakida, and M. Suzuki, "Finite-element analysis of edge resonance in a semi-infinite elastic plate," Electron. Lett., vol. 19 , pp. 256-257, 1983.

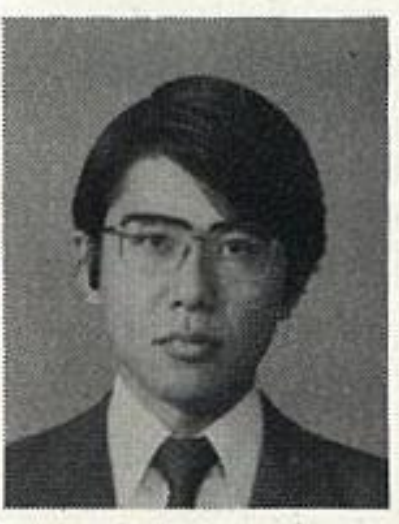

Masanori Koshiba was born in Sapporo, Japan, on November 23, 1948. He received the B.S., M.S., and Ph.D. degrees in electronic engineering from Hokkaido University, Sapporo, Japan, in 1971,1973 and 1976 , respectively.

In 1976 he joined the Department of Electronic Engineering, Kitami Institute of Technology, Kitami, Japan. Since 1979 he has been an Assistant Professor of Electronic Engineering at Hokkaido University. He has been engaged in research on surface acoustic waves, dielectric optical waveguides, and waveguide discontinuities.

Dr. Koshiba is a member of the Institute of Electronics and Communication Engineers of Japan. 


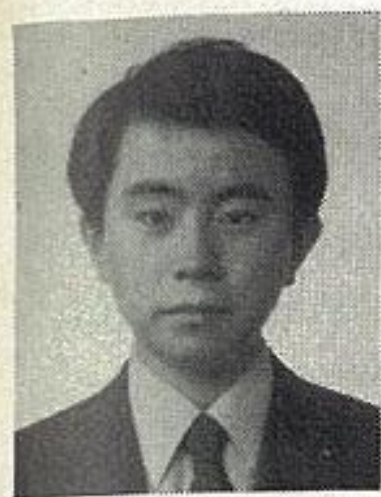

Shoji Karakida was born in Koshoku, Japan, on March 4, 1959. He received the B.S. degree in electronic engineering from Hokkaido University, Sapporo, Japan, in 1982.

$\mathrm{He}$ is presently studying toward the M.S. degree in electronic engineering, Hokkaido University, Sapporo, Japan.

Mr. Karakida is a member of the Institute of Electronics and Communication Engineers of Japan.

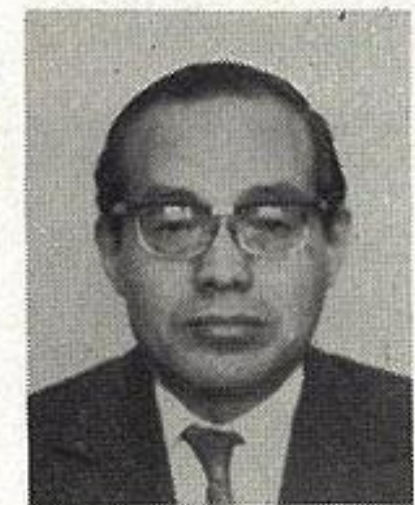

Michio Suzuki (SM'57) was born in Sapporo, Japan, on November 14, 1923. He received the B.S. and Ph.D. degrees in electrical engineering from Hokkaido University, Sapporo, Japan, in 1946 and 1960 , respectively.

From 1948 to 1962 he was an Assistant Professor of Electrical Engineering at Hokkaido University. Since 1962 he has been a Professor of Electronic Engineering at Hokkaido University. From 1956 to 1957 he was a Research Associate at the Microwave Research Institute of Polytechnic Institute of Brooklyn, Brooklyn, NY.

Dr. Suzuki is a member of the Institute of Electronics and Communication Engineers of Japan, the Institute of Electrical Engineers of Japan and the Institute of Television Engineers of Japan. 